\title{
Serial left-ventricular biopsy sampling using a minimally invasive trans-thoracic approach in adult dogs
}

\author{
Dirk W. Donker • Jos G. Maessen • \\ Roel L. H. M. G. Spätjens • Theo van der Nagel • \\ Monique de Jong • Frans C. Ramaekers • \\ Harry J. G. M. Crijns • Marc A. Vos • \\ Paul G. A. Volders
}

Received: 7 February 2007 / Accepted: 21 March 2007 /Published online: 16 May 2007

(C) Springer-Verlag 2007

\begin{abstract}
Myocardial biopsies are an increasingly important tool to unravel the molecular mechanisms of cardiac disease. We evaluate a novel minimally invasive transthoracic approach for left-ventricular (LV) intra-mural biopsies, which enables repetitive individual sampling in adult dogs. Forty three generally anaesthesised dogs were studied during sinus rhythm (SR, control) and multiple times after the induction of volume overload hypertrophy (complete atrioventricular block [AVB]). Through a small cutaneous incision, an automatic biopsy needle was advanced into the apicolateral LV, guided by fluoroscopy. Electrocardiography (ECG), LV pressure and echocardiography served to monitor the procedure. One hundred
\end{abstract}

D. W. Donker • R. L. H. M. G. Spätjens • M. de Jong •

H. J. G. M. Crijns • P. G. A. Volders $(\bowtie)$

Department of Cardiology,

Cardiovascular Research Institute Maastricht,

Academic Hospital Maastricht and Maastricht University,

P.O. Box 5800, 6202 AZ Maastricht,

The Netherlands

e-mail: p.volders@cardio.unimaas.nl

J. G. Maessen · T. van der Nagel • M. de Jong

Department of Cardiothoracic Surgery,

Cardiovascular Research Institute Maastricht,

Academic Hospital Maastricht and Maastricht University,

Maastricht, The Netherlands

F. C. Ramaekers

Department of Molecular Cell Biology,

Cardiovascular Research Institute Maastricht,

Maastricht University,

Maastricht, The Netherlands

M. A. Vos

Department of Medical Physiology,

University Medical Center Utrecht,

Utrecht, The Netherlands eighty-eight intra-mural LV biopsies were obtained in 82 serial experiments (usually SR, 1, 2 and 6 weeks AVB) with a maximum of 8 repeated biopsies. All biopsies $\left(\sim 10 \mathrm{~mm}^{3}\right)$ were suitable for simultaneous application of different cell-biological (light and electron microscopy, immunohistochemistry) and molecular techniques (PCR, Western blotting). In chronic experiments, repeated biopsy sampling did not influence haemodynamics, mechanics, electrocardiographic parameters or myocardial remodelling during SR or AVB. The rate of significant complications was as low as $4 \%$ of experiments. Minimally invasive sampling of LV needle biopsies enables serial assessment of myocardial remodelling using different molecular techniques in individual animals. The technique is safe and has no long-term effects on cardiac function or structure.

Keywords Instruments and techniques · Myocardial biopsy · Myocardial remodelling · Ventricles

\section{Introduction}

Myocardial biopsy sampling offers indispensable diagnostic options to unravel the mechanisms of myocardial disease in different conditions [3, 18]. Currently, cardiac biopsies are mostly obtained using a trans-venous catheter-based bioptome enabling to sample sub-endomyocardial tissue from the right ventricle (RV), as introduced in 1962 [13]. This trans-vascular approach is also used trans-arterially to sample from the left ventricle (LV) [9]. In experienced hands, the catheter-based technique is relatively safe with complication rates of $1-4 \%$ [10] and is routinely applied in clinical referral centers [9]. It is important to note that the limited invasiveness of the technique makes it possible to 
a

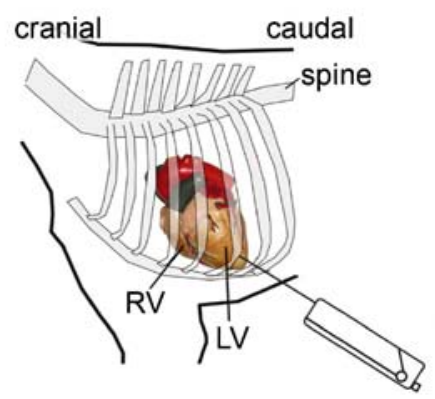

C

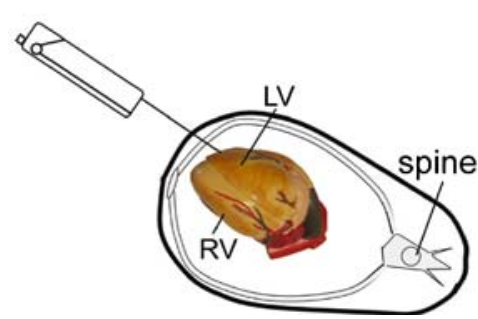

Fig. 1 Serial percutaneous sampling of LV intra-mural biopsies. a Schematic illustration of optimal biopsy needle positioning in the sagittal plane in relation to the intra-thoracic location of the heart in the adult dog. b Under fluoroscopic guidance, the needle is advanced into the apicolateral LV wall. c Schematic illustration of optimal biopsy needle positioning in the transverse plane (caudal view) in relation to the intra-thoracic location of the heart. d A small incision facilitates percutaneous introduction of the biopsy needle into the left anterolateral inter-costal space overlying the LV apex. See text for detailed description

sample cardiac biopsies in a serial manner to monitor specific disease processes in individual patients over time. Yet, sub-endomyocardial tissue samples have significant drawbacks relating to (1) sampling errors [15] and lack of representativity, which requires to take at least 5 biopsies per session and even more for focal processes [19]; (2)
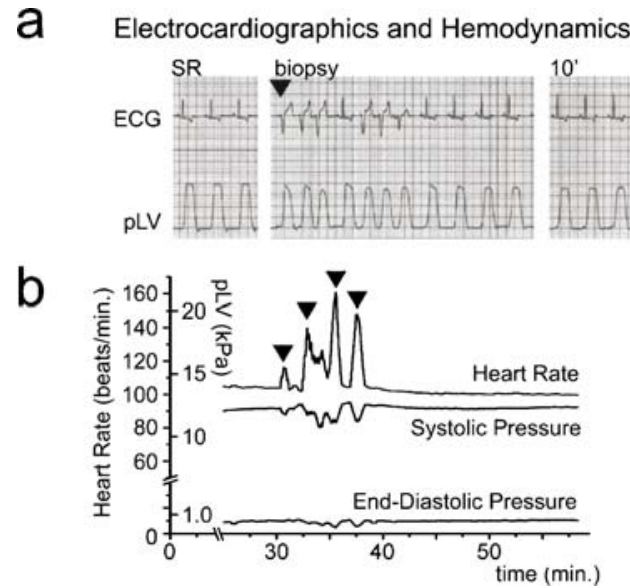

Fig. 2 Procedural monitoring during biopsy sampling. a Electrocardiographic and haemodynamic monitoring shows ventricular extrasystoles (arrowhead) during intra-myocardial insertion of the biopsy needle. $S R$, sinus rhythm, $p L V$, intra-cavitary $L V$ pressure. Ten minutes after sampling, the ECG and LV pressure tracings are similar to the baseline (SR). b Monitoring of heart rate and LV systolic and diastolic pressure trends reveal stable tracings after sequential sampling of four small sample size (usually $<1 \mathrm{~mm}^{3}$ ) [15] and (3) poor tissue quality due to squeezing and cutting artefacts in a relatively small tissue sample [11], which often render the biopsies unsuitable for histology, immunohistochemistry and electron microscopy [15].

An alternative way to sample ventricular myocardial tissue is by the use of a biopsy needle, which enables to obtain larger, intra-mural samples of superior quality compared to endomyocardial biopsies. This technique was first described using limited thoracotomy [7], but needle biopsies have generally been confined to the use in patients and animals undergoing standard thoracotomy for direct vision of the puncture site and potential bleeding [17]. Today, needle biopsies can be safely and quickly obtained during open-heart surgery using automatic biopsy devices [14, 20].

In the study reported here, we developed a novel transthoracic approach to sample LV needle biopsies from individual anaesthetised dogs in a serial manner and evaluated its application in chronic experiments after the induction of cardiac hypertrophy.

\section{Materials and methods}

Experiments were conducted in accordance with the 'European Directive for the Protection of Vertebrate Animals Used for Experimental and Other Scientific Purposes (86/609/ EU)'. The Committee for Experiments on Animals of Maastricht University approved the experiments.

Serial trans-thoracic sampling of LV intra-mural biopsies

We studied a total number of 43 adult mongrel dogs of either gender weighing $28 \pm 1 \mathrm{~kg}$ at baseline during normal sinus

C Echocardiography
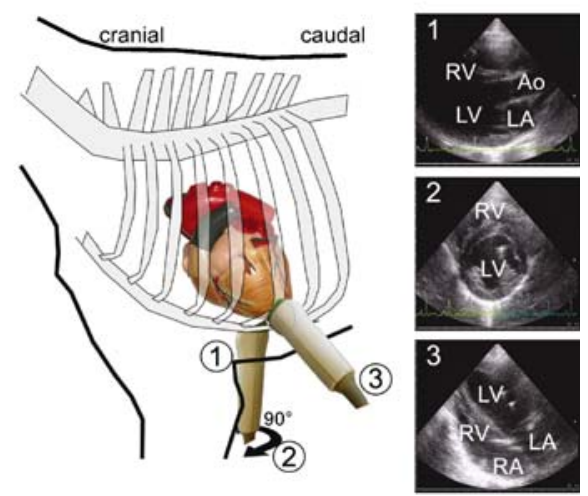

biopsies accompanied by multiple ventricular extra-systoles (arrowheads). c Trans-thoracic echocardiographic approach in the adult dog. The right parasternal transducer positions allows long axis (1) and, after rotation, short axis views (2), whereas left parasternal apical scanning provides four-chamber views (3). Note that besides B-mode also M-mode and Doppler-flow measurements (not shown) can be easily performed. $A o$, ascending aorta; $L A$, left atrium; $R A$, right atrium 
rhythm (SR, control). All experiments were performed under general anaesthesia. After overnight fasting, pre-medication $(0.15 \mathrm{mg} / \mathrm{kg}$ acepromazine, $0.4 \mathrm{mg} / \mathrm{kg}$ methadone, $0.06 \mathrm{mg} /$ $\mathrm{kg}$ atropine i.m.) was administered. Complete anaesthesia was induced by thiopental $(20 \mathrm{mg} / \mathrm{kg}$ i.v. $)$ and maintained with halothane $(0.5-1 \%)$ and $\mathrm{O}_{2}: \mathrm{N}_{2} \mathrm{O}(1: 2)$.

The dogs were positioned in a right-sided supine position, the left anterolateral thorax was shaved, disinfected with iodine and draped with sterile towels. The apex was palpated on the left-sided chest. The X-ray tube was set to a vertical position $\left(0^{\circ}\right)$ to identify the apicolateral $\mathrm{LV}$ in the sagittal plane (Fig. 1a), and visualised in an oblique leftlateral fluoroscopic view (Fig. 1b). In the sagittal plane, the biopsy needle forms an angle of $\sim 60^{\circ}$ with the longitudinal axis of the sternum. In the transverse plane (Fig. 1c), the apicolateral LV was approached by holding the biopsy needle in an angle of $\sim 60^{\circ}$ to an imaginary horizontal line, in parallel to the table (Fig. 1c). Mechanical ventilation was briefly stopped during sampling to minimise the risk of pulmonary injury. A $14 \mathrm{G}$ automatic biopsy needle (ACECUT, TSK Laboratory, Japan) was opened and slowly advanced trans-thoracically through a small $(\sim 5 \mathrm{~mm})$ cutaneous incision in the inter-costal space overlying the LV apex (Fig. 1d). This was done under continuous fluoroscopic guidance whilst approaching the apicolateral LV wall (Fig. 1b). Contact with the epicardial surface was recognised by sensing a mild resistance and cardiac pulsations as pulse-synchronous motion of the needle shaft, often accompanied by ventricular extra-systoles on the electrocardiogram (ECG) (Fig. 2a). Subsequently, the needle was quickly advanced into the epicardial layers at an angle of $\sim 60^{\circ}$ to the surface. Then, it was forwarded by $\sim 10 \mathrm{~mm}$ as controlled by centimetre markers on the shaft to assure deep intra-mural sampling. The oblique angle to
Fig. 3 Processing of LV myocardial needle biopsies. a Biopsies can be easily lifted from the needle with tweezers. The specimen can be processed as a complete intra-mural cylinder or can be dissected into smaller pieces. After freezing, cryosections can be prepared, allowing simultaneous application of different analytical methods as e.g. Histo histology, IHC immunohistochemistry, $E M$ electron microscopy, $P C R$ polymerase chain reaction and $W B$ Western blotting. Scale bar indicates $1 \mathrm{~cm}$. b The LM photomicrograph illustrates the good quality of the myocardial samples using Masson's Trichrome staining (cardiomyocytes $[\mathrm{red}] /$ fibrous tissue [blue]). This example of an intra-mural biopsy extends from the epicardium (top inset) to the endocardium (bottom inset). Scale bars indicate $1 \mathrm{~mm}$. c Intra-mural extension of the biopsy sample can also be confirmed by visualisation of the (sub)endocardial layers as illustrated in the light-microscopic photograph using toluidine blue counterstained with periodic acid Schiff; endocardium (pale blue), working myocardium (blue) and glycogen (magenta)containing Purkinje fibres. Scale bars indicate $100 \mu \mathrm{m}$. d At autopsy, only limited scar formation occurred at the sampling sites (arrowheads) after repeated biopsies. Scale bar indicates $2 \mathrm{~mm}$
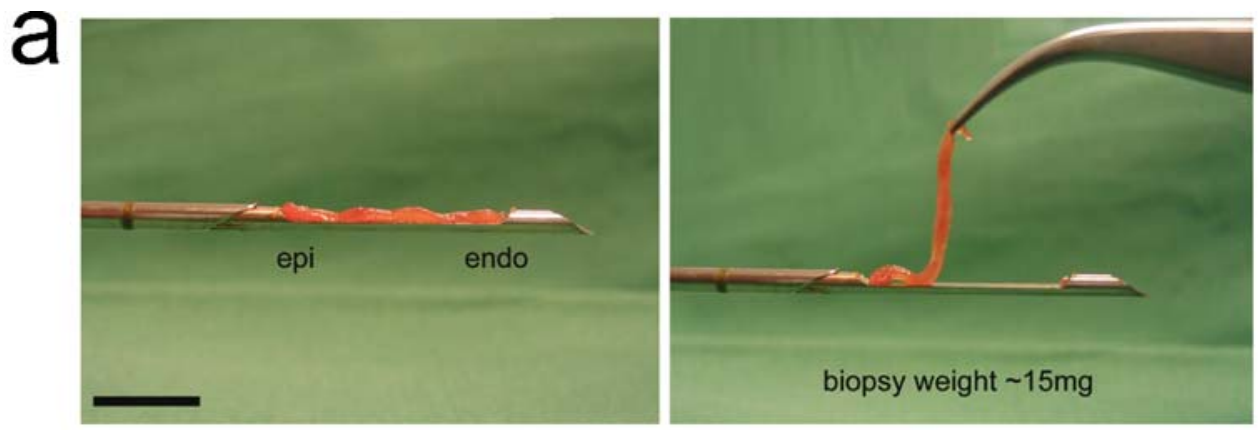

\section{(cryo)sections}

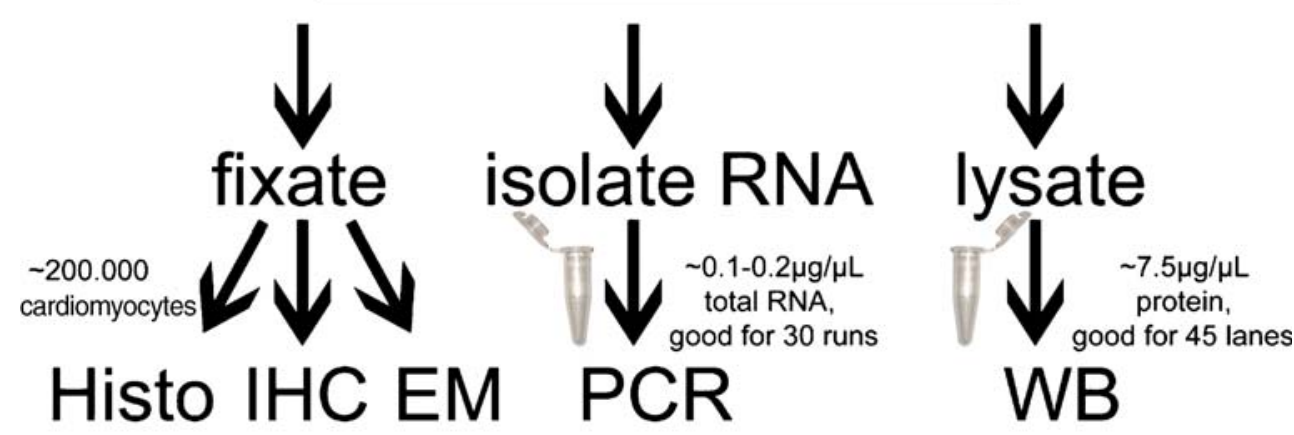

b

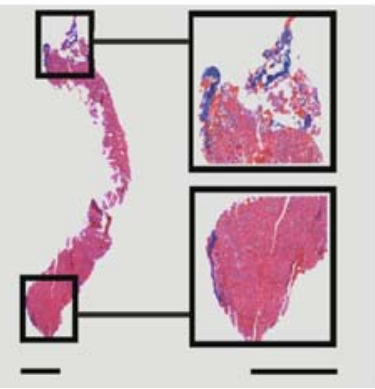

c

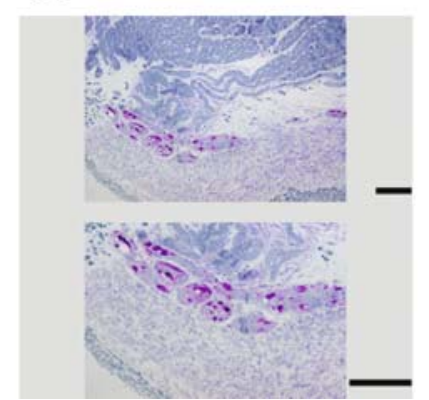

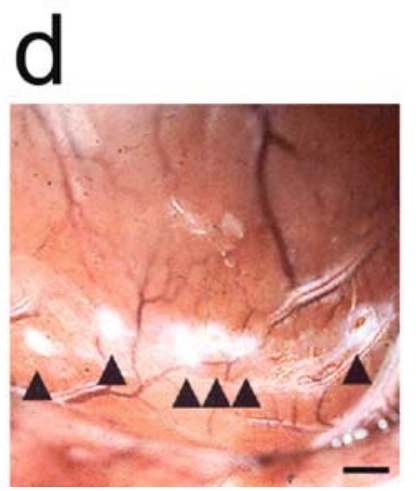


the epicardial surface was applied to minimise the risk of haemopericardium due to trans-mural puncture. After puncture, the automatic cutting mechanism of the biopsy needle was immediately released and the device was retracted. We aimed at completing the intra-myocardial insertion of the needle, the actual sampling and the retraction of the device within 2-3 heartbeats to avoid unnecessary movements of the device, potentially causing additional damage or bleeding of the myocardium or arrhythmias. At least two cylindrical transmural samples were taken per experiment. For further processing, biopsies were either immediately frozen in isopentane, pre-cooled with liquid nitrogen and stored at $-80^{\circ} \mathrm{C}$ or fixed in $3 \%$ glutaraldehyde and stored at $4{ }^{\circ} \mathrm{C}$. Light and electron microscopy, PCR and Western blotting were performed according to standard protocols $[4,16]$.

Throughout the procedure, a standard 6-lead ECG was registered and the LV intra-cavitary blood pressure was monitored in a sub-set of experiments (Fig. 2a and Fig. 2b). After sampling, all animals were followed-up echocardiographically (Fig. 2c) for at least $60 \mathrm{~min}$ to monitor LV contractility and potential occurrence of pericardial effusion. Echocardiograms were usually performed every $5 \mathrm{~min}$ during the first $20 \mathrm{~min}$ after sampling. If pericardial effusion was absent or had not increased to a maximum of more than $\sim 5 \mathrm{~mm}$, anaesthesia was terminated. After detubation, additional echocardiograms were performed before and after transportation to the cage, usually at 30 , 45 and $60 \mathrm{~min}$ after sampling. When pericardial effusion increased to more than $\sim 10 \mathrm{~mm}$ in the first hour after sampling, we decided to intervene surgically by left-lateral thoracotomy, pericardiotomy, evacuation of blood from the pericardial space and local haemostasis of the bleeding site. LV haemodynamics, mechanics and echocardiographic estimates of LV mass were assessed as described before $[4,5]$. The complete procedure is described as a practical step-by-step approach in Flowchart 1.

All dogs were studied serially during SR and after the induction of complete atrioventricular block (AVB). AVB was induced by radiofrequency (RF) catheter ablation of the His bundle. Sampling was performed under baseline conditions at sinus rhythm (SR; control) and, in the same animals, at different time points after AVB ranging from 2 days up to 6 weeks. The dogs were sacrificed several weeks later and the hearts were excised and inspected macroscopically.
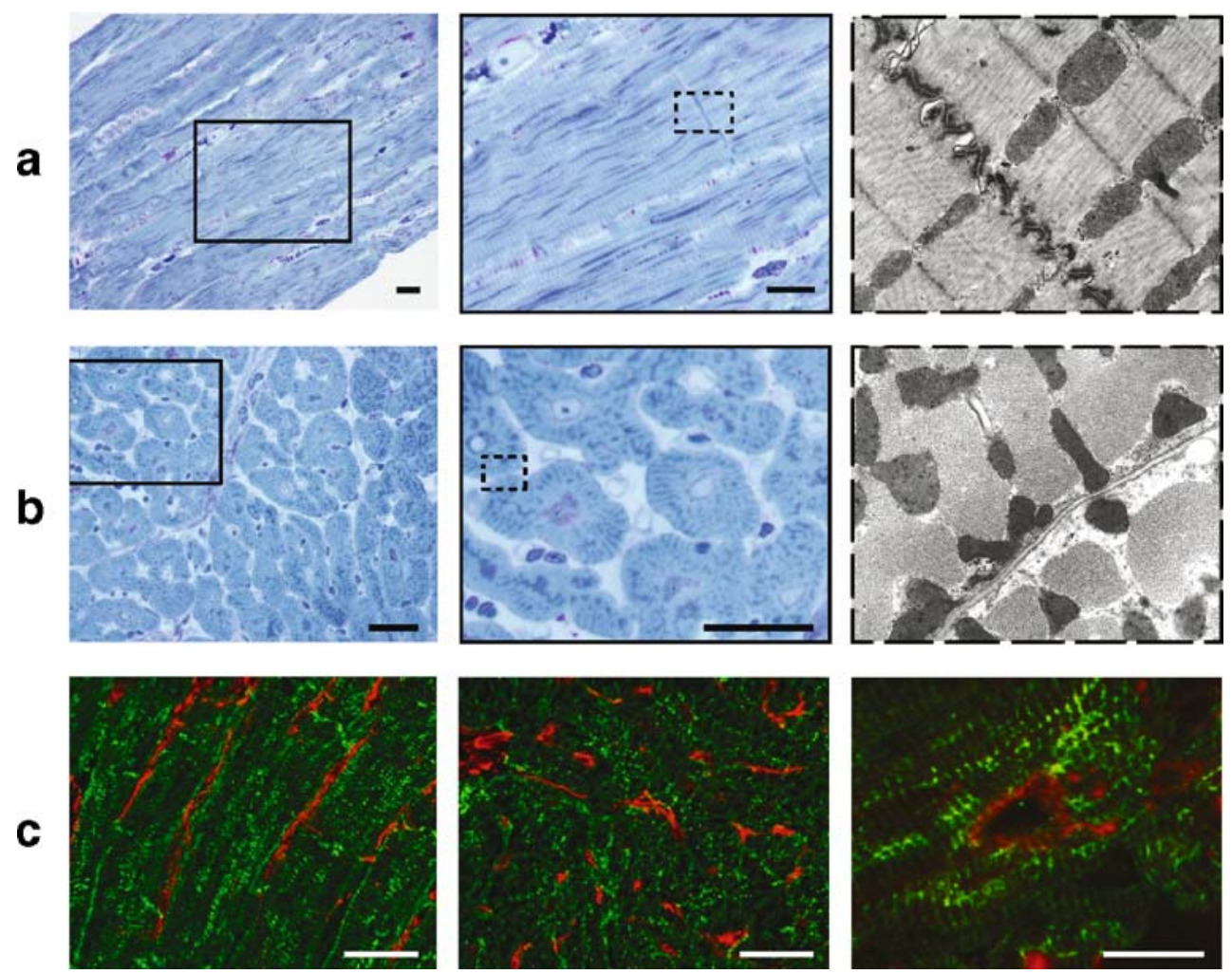

Fig. 4 Cell-biological myocardial analysis in LV needle biopsies. a Representative light-microscopic (toluidine blue counterstained with periodic acid Schiff) and electron-microscopic photographs showing longitudinally sectioned cardiomyocytes within a normal myocardial texture from a dog bioptised during SR, demonstrating the excellent tissue quality. Scale bars indicate $20 \mu \mathrm{m}$. b Light-microscopic and electron-microscopic photographs corresponding to a showing cross-

sectioned cardiomyocytes. Scale bars indicate $20 \mu \mathrm{m}$. c Immunofluorescent histochemistry in LV myocardium double-labelling the cardiomyocyte intermediate filament desmin (green), the fibroblast intermediate filament vimentin (red; left and middle) and alpha smooth muscle actin present in the vessel wall (red; right), in longitudinally (left, right) and transversally sectioned myocardium (middle). Scale bars indicate $20 \mu \mathrm{m}$ 


\section{Statistics}

Data are presented as the mean \pm standard error of the mean (SEM). Data were compared using Student's $t$-test for unpaired data. Differences were considered statistically significant if $P<0.05$.

\section{Results}

In 82 experiments, a total number of 188 intra-mural LV biopsies was obtained ( $2.6 \pm 0.6$ biopsies per experiment). In individual animals, up to 4 consecutive experiments were conducted in which maximally 8 repeated biopsies were taken, typically at SR, 1, 2 and 6 weeks of AVB.

The biopsies measured $\sim 1 \times 1 \times 10 \mathrm{~mm}$ and weighed $\sim 15 \mathrm{mg}$ (Fig. 3a). The tissue was of good quality and suitable for subsequent analysis in all biopsies. To assess the intra-mural extension of the biopsies, we performed lightmicroscopic analyses of complete specimens (Fig. 3b) and (sub)endocardial pieces (Fig. 3c), demonstrating that (sub)

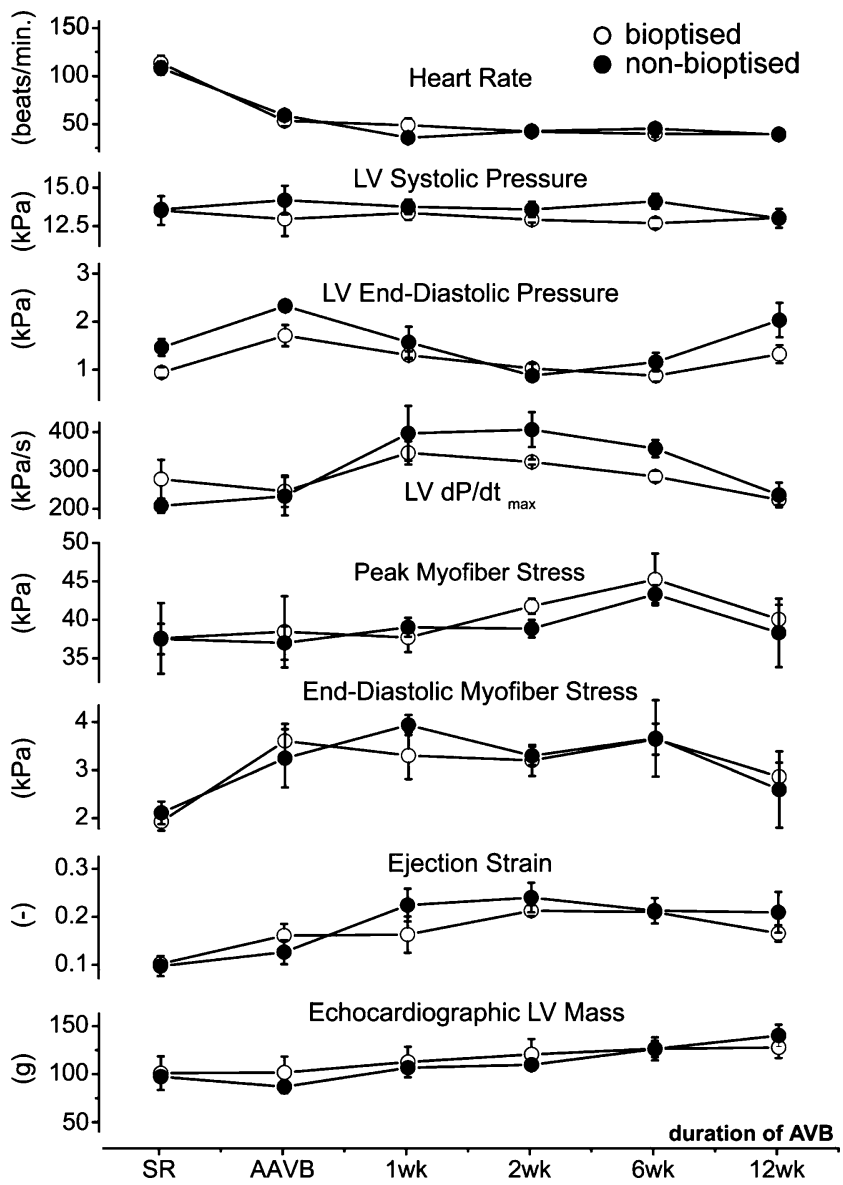

Fig. 5 Serial analysis of LV haemodynamics, mechanics and hypertrophy in bioptised vs non-bioptised animals. Group mean values of 4 dogs bioptised at SR, 1, 2 and 6 weeks AVB compared to 4 non-bioptised dogs at identical experimental time points. $P=\mathrm{NS}$ for any parameter between the two groups epicardial and (sub)endocardial myocardium is present in, at least, a sub-set of the biopsies. Macroscopic inspection of the sampling sites revealed proper sampling from the apicolateral LV wall and only minimal local fibrosis with a diameter of $\sim 1-2 \mathrm{~mm}$ limited to the puncture site (Fig. 3d).

Light and electron microscopy revealed a normal homogeneous myocardial texture in the absence of contraction bands allowing detailed analyses of the cardiomyocyte (ultra) structure and of cell-cell contacts (Fig. 4a and Fig. 4b). Representative immunohistochemical double labelling allows to study intra-myocardial protein localisation and their spatial distribution pattern in cardiomyocytes, noncardiomyocytes and extra-cellular matrix (Fig. 4c).

Serial LV function and structure in bioptised vs non-bioptised dogs

Serial analysis of LV systolic and diastolic haemodynamics, mechanics and LV mass increase in chronic experiments revealed no significant differences between repeatedly bioptised and non-bioptised individual dogs after induction of bradycardia-induced volume overload due to AVB (Fig. 5). Also, electrocardiographic parameters were comparable in both groups over time (Fig. 6).

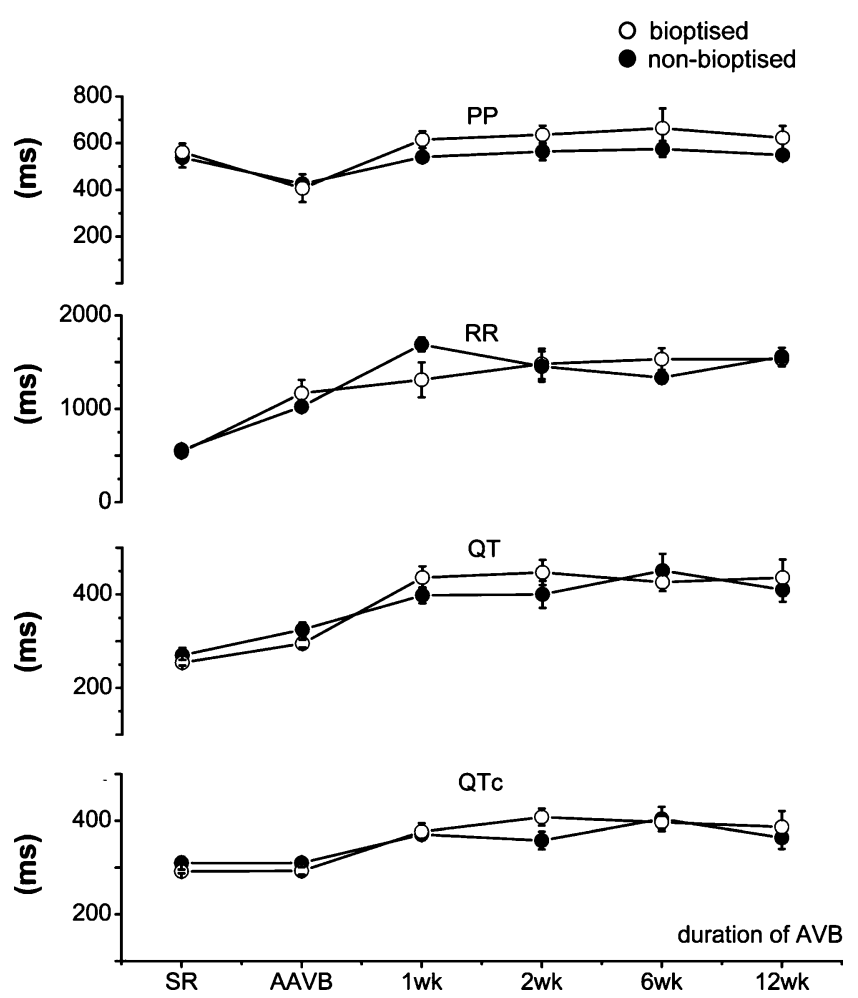

Fig. 6 Serial analysis of electrocardiographic parameters in bioptised vs non-bioptised animals. Group mean values of 4 dogs bioptised at SR, 1, 2 and 6 weeks AVB compared to 4 non-bioptised dogs at similar experimental time points. $P=\mathrm{NS}$ for any parameter between the two groups 
To control for influences of repeated biopsy sampling itself on protein expression, we studied cardiomyocytespecific proteins known to change after AVB, such as the $\mathrm{K}^{+}$channel sub-unit KCNQ1 and the $\beta-1$ adrenergic receptor [16]. The expression level of both proteins remained unaltered in repeated biopsies of three control dogs (Fig. 7).

Procedural experiences and complications

Generally, the animals recovered from the procedure within a few hours and post-operative wound infection was not observed. In the first 34 experiments, we observed cardiac tamponade in 5 (15\%) experiments. Four dogs died (12\%) and 1 underwent successful left-lateral thoracotomy, pericardiotomy and operative evacuation of the haemopericardium. It is important to note that all these dogs were heparinised (1,000 IU i.v.) around biopsy sampling, as routinely applied in our operation room to avoid catheterinduced thromboembolism and only 3 dogs had been haemodynamically monitored during the experiment.

To reduce the initial complication rate, we adapted the procedure: heparin was omitted without negative sequelae, and echocardiography was introduced to monitor the procedure, especially the occurrence of haemopericardium. The latter occurred in only 2 (4\%) of 48 experiments and could be immediately diagnosed and treated surgically in 1 dog having tamponade, whereas the other dog was found dead in the cage $>12 \mathrm{~h}$ after the experiment. Autopsy revealed a haemopericardium due to puncture of a small coronary side-branch. We summarised our experiences in Table 1.

\section{Discussion}

In the present study, we demonstrate how LV needle biopsies can be repeatedly obtained in dogs undergoing chronic experiments. A trans-thoracic approach together with optimal safety monitoring ensures a low complication rate, quick post-operative recovery and optimal tissue quality.

Serial myocardial sampling using needle biopsies in a chronic experimental setting is generally limited by the invasiveness of a thoracotomy, which results in only few sampling points, e.g., at the control stage and before sacrifice of the dogs [17]. We circumvented this by choosing a trans-thoracic, percutaneous approach, thus avoiding the thoracotomy. Historically, different closedchest approaches have been reported in canine and human hearts to sample ventricular needle biopsies from the LV anterior wall, apical area [2] and the inter-ventricular septum [1] under local or general anaesthesia. After the 1970s, percutaneous needle biopsy techniques have not been widely applied in patients or experimental animals, and no further technical developments have been reported. This might be largely due to the advent of catheter-based techniques, which are less invasive, relatively easy to use and safe, and, moreover, suitable for repeated biopsies.

The novel trans-thoracic approach we described in this study shares many advantages with the trans-vascular technique: (1) it is minimally invasive; (2) it has a low rate of potentially life-threatening complications ( $4 \%)$ despite the limited number of experiments we performed, which is comparable to the clinically accepted transvascular techniques [10]; (3) it is suitable for repeated biopsies and thereby allows individual follow-up studies; (4) it can be performed within a few minutes; (5) it can be easily performed in a standard experimental animal laboratory (see Flowchart 1) with experienced bio-technicians reaching a sufficient level of competence after having performed $\sim 25$ biopsies. Moreover, the approach can likely be applied in other large animal species. This assumption is based on comparative anatomical considerations and reports of experiments with minimally invasive thoracoscopic cardiac access in sheep [6], pigs [21] and goats.
Fig. 7 Serial analysis of myocardial protein expression before and after biopsy sampling. a Western blot analysis shows that the $\mathrm{K}^{+}$-channel subunit KCNQ1 and $\mathbf{b}$ the $\beta-1$ adrenergic receptor remained unaltered in repeated biopsies of control dogs in SR
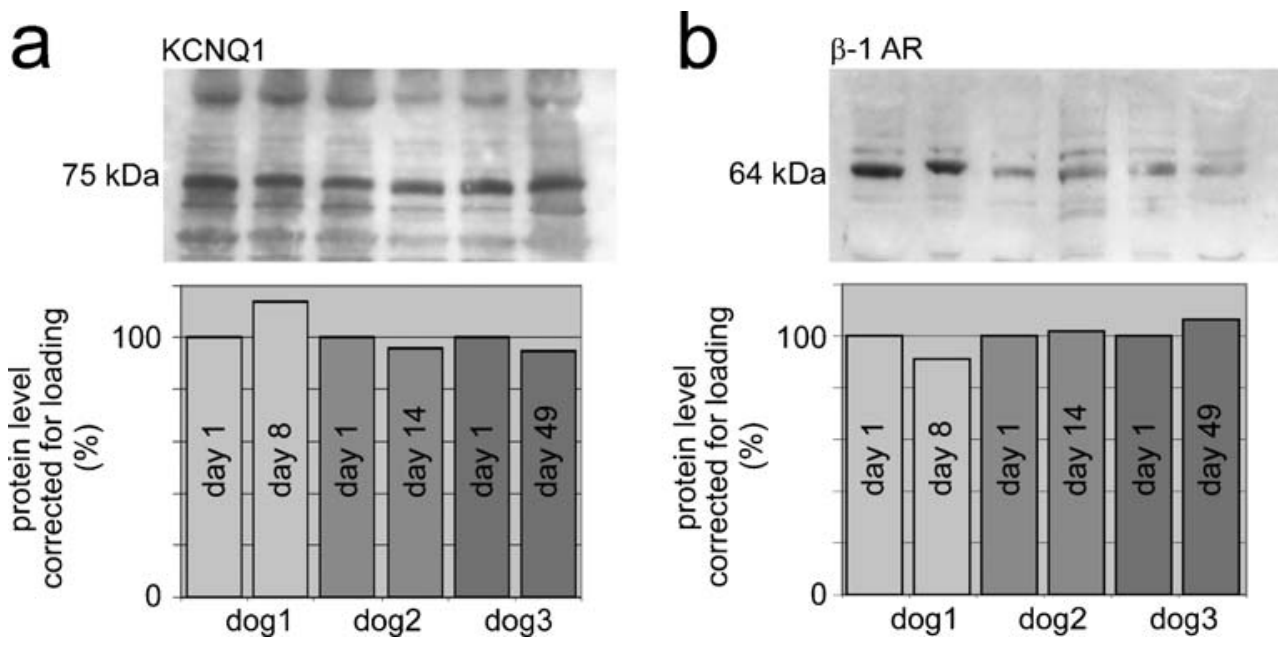
Table 1 Practical considerations

\begin{tabular}{|c|c|c|c|}
\hline Parameter & Technique & Recommendation & $\begin{array}{l}\text { Complications/ } \\
\text { comments }\end{array}$ \\
\hline $\begin{array}{l}\text { Identification } \\
\text { of sampling } \\
\text { site }\end{array}$ & $\begin{array}{l}\text { Palpation/ } \\
\text { fluoroscopy }\end{array}$ & $\begin{array}{l}\text { Sample in apicolateral LV, avoid thin-walled apex and } \\
\text { coronaries in the lateral LV }\end{array}$ & $\begin{array}{l}\text { Cardiac tamponade, coronary } \\
\text { perforation }\end{array}$ \\
\hline \multirow{3}{*}{$\begin{array}{l}\text { Intra- } \\
\text { myocardial } \\
\text { sampling }\end{array}$} & Operator & $\begin{array}{l}\text { Feel mild resistance and cardiac pulsations on needle shaft } \\
\text { before intra-myocardial insertion }\end{array}$ & Extra-myocardial biopsy, no biopsy \\
\hline & ECG & $\begin{array}{l}\text { Monitor the occurrence of ventricular extra-systoles } \\
\text { originating from the biopsy site }\end{array}$ & Extra-myocardial biopsy, no biopsy \\
\hline & Microscopy & $\begin{array}{l}\text { Use standard histological staining to ensure tissue quality and } \\
\text { intra-myocardial extent, e.g. haematoxylin/eosin, toluidin } \\
\text { blue, Masson's trichrome }\end{array}$ & $\begin{array}{l}\text { Light microscopy allows to visualise } \\
\text { endocardium/epicardium and } \\
\text { myocardium in the same section }\end{array}$ \\
\hline \multirow[t]{3}{*}{$\begin{array}{l}\text { Cardiac } \\
\text { function }\end{array}$} & ECG & Monitor standard leads throughout the experiment & $\begin{array}{l}\text { Sinus tachycardia and signs of } \\
\text { ischaemia can aid to diagnose a } \\
\text { cardiac tamponade in time }\end{array}$ \\
\hline & Echocardiography & $\begin{array}{l}\text { Assess cardiac contractility and pericardial effusion directly } \\
\text { after sampling and repeat frequently in the first hour }\end{array}$ & $\begin{array}{l}\text { Cardiac tamponade can be diagnosed in } \\
\text { time }\end{array}$ \\
\hline & $\begin{array}{l}\text { LV intra-cavitary } \\
\text { pressure catheter } \\
\text { (optional) }\end{array}$ & $\begin{array}{l}\text { Monitor LV systolic blood pressure throughout the experiment } \\
\text { (optional) }\end{array}$ & $\begin{array}{l}\text { Progressive decrease of systolic LV } \\
\text { blood pressure can aid to diagnose a } \\
\text { cardiac tamponade in time }\end{array}$ \\
\hline
\end{tabular}

${ }^{a}$ Arrhythmic ventricular responses may incidentally be absent.

$L V$ : left ventricle

Multiple serial intra-mural biopsies from the same LV area did not influence functional and structural remodelling processes that occur after AVB. All analysed mechanical parameters were compatible with our earlier work $[4,5]$. Also, under control conditions, repeated biopsy sampling from the apicolateral LV wall did not significantly influence the expression of cardiomyocyte-specific proteins that have been shown to be altered in remodelling processes early after the induction of AVB [16]. These findings support the minimally invasive character of the technique and are in line with the macroscopic finding of only slight local fibrosis at barely visible puncture sites. Thus, it can be concluded that myocardial damage is confined to the puncture site. However, the total number of biopsies taken in individual animals might be relevant in this regard. We obtained a maximum of eight serial needle biopsies in up to four consecutive closed-chest experiments per dog. Higher numbers of serial ventricular needle biopsies in chronic experiments have only been reported in studies using cardiac allograft biopsies in dogs undergoing orthotopic [12] or heterotopic [8] heart transplantation. In these studies, post-operative fibrosis and adhesions after open-heart surgery likely play a role in reducing the risk of haemopericardium and tamponade, but may also increase the probability of biopsy-induced functional and structural myocardial changes.

Needle biopsies offer ample opportunities for a detailed myocardial analysis (Fig. 3, Fig. 4 and Fig. 7). The tissue specimens are relatively large, cutting artefacts are limited to the border zones and they represent a cross-section through the ventricular wall potentially extending into the (sub)endocardium (Fig. 3). Our serial approach allows to discern even mildly fluctuating expression patterns in longterm studies because individual dogs serve as their own controls $[4,16]$. These subtle changes are likely missed in group comparisons due to inherent inter-individual differences. These aspects meet contemporary issues in cardiac research, aimed at a comprehensive multi-level molecular analysis in individual subjects.

\section{Conclusions}

A novel minimally invasive trans-thoracic approach for serial LV intra-mural needle biopsies has been successfully developed in adult dogs. The procedure is safe and allows repeated sampling in individual animals without influencing cardiac function or structure in long-term experiments. Different cell-biological and molecular techniques can be simultaneously applied in these biopsy specimens. The approach is potentially applicable to other animal models of cardiac disease and offers the opportunity to study molecular mechanisms relevant for myocardial remodelling in chronic experiments relating to various pathological conditions.

Acknowledgments This study was financially supported by the 'Stichting Hartsvrienden Rescar', Maastricht, The Netherlands. Dirk W. Donker was supported by Medtronic, The Netherlands. The authors wish to thank Jet Beekman (Dept. of Med. Physiol., Utrecht), Helma Kuijpers and Fons Verheyen (Dept. of Mol. Cell Biol., Maastricht) for technical assistance. 


\section{Appendix}

Flowchart 1 Procedure-minimally invasive biopsy technique

Induce general anesthesia, intubate and start mechanical ventilation

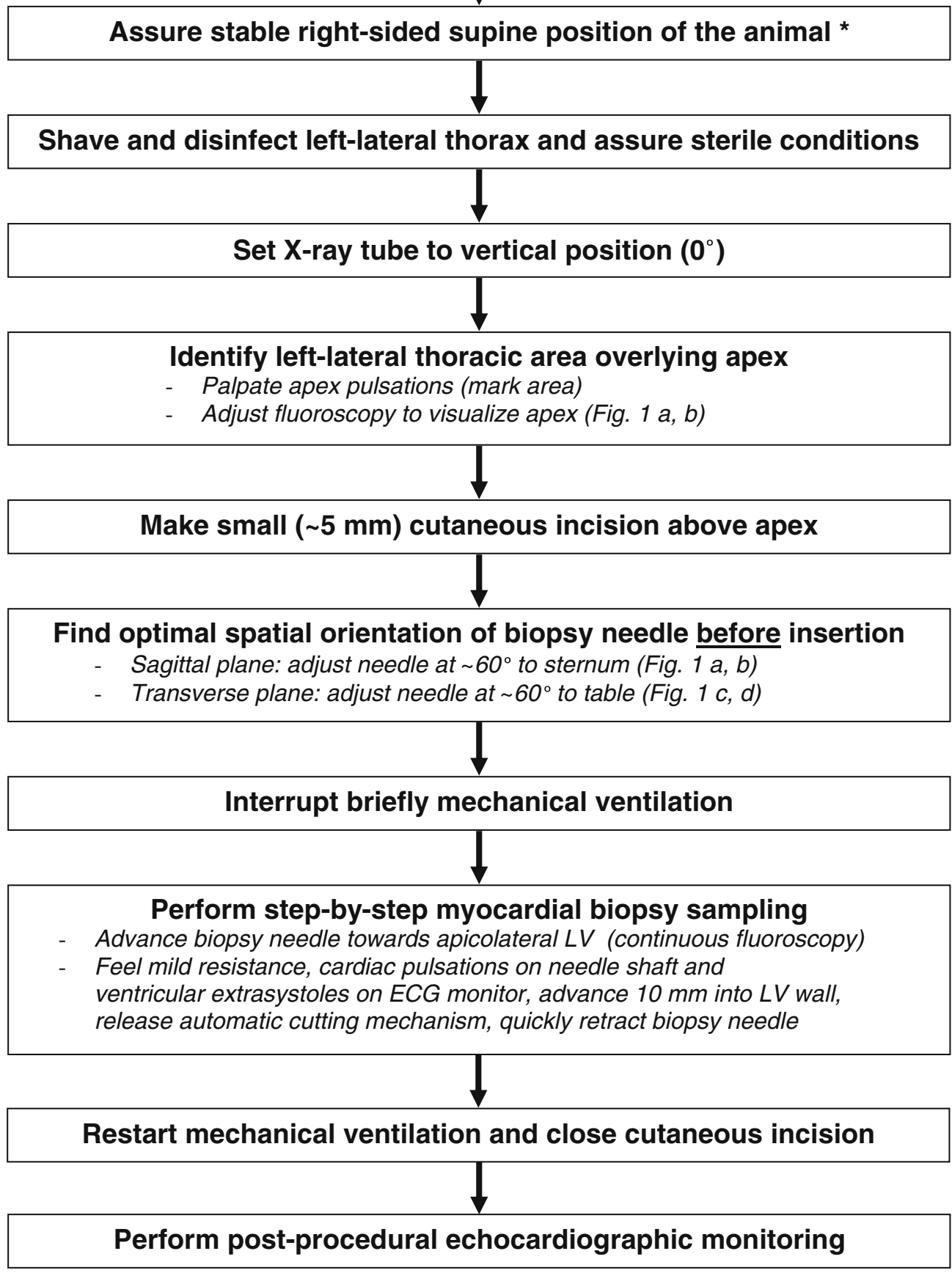

* Perform baseline echocardiograms for comparison to post-procedural echocardiograms 


\section{References}

1. Bercu B, Heinz J, Choudhry AS, Cabrera P (1964) Myocardial biopsy. A new technic utilizing the ventricular septum. Am J Cardiol 14:675-678

2. Casten GG, Marsh JB (1953) Metabolic studies on cardiac tissue obtained by needle biopsy in the intact unanesthetized dog. Circ Res 1:226-229

3. Cunningham KS, Veinot JP, Butany J (2006) An approach to endomyocardial biopsy interpretation. J Clin Pathol 59:121-129

4. Donker DW, Maessen JG, Verheyen F, Ramaekers FC, Spätjens RLHMG, Kuijpers H, Ramakers C, Schiffers PMH, Vos MA, Crijns HJGM, Volders PGA (2007) Impact of acute and enduring volume overload on mechanotransduction and cytoskeletal integrity of canine left-ventricular myocardium. Am J Physiol Heart Circ Physiol 292:H2324-H2332 DOI 10.1152/ajpheart.00392.2006

5. Donker DW, Volders PGA, Arts T, Bekkers BCAM, Hofstra L, Spätjens RLHMG, Beekman JD, Borgers M, Crijns HJGM, Vos MA (2005) End-diastolic myofiber stress and ejection strain increase with ventricular volume overload - serial in-vivo analyses in dogs with complete atrioventricular block. Basic Res Cardiol 100:372-382

6. Joudinaud TM, Kegel CL, Gabster AA, Sanz ML, MacDonald A, Propp D, Callaghan E, Weber PA, Hvass U, Duran CM (2005) An experimental method for the percutaneous induction of a posterolateral infarct and functional ischemic mitral regurgitation. J Heart Valve Dis 14:460-466

7. Kent G, Sutton DC, Sutton GC (1956) Needle biopsy of the human ventricular myocardium. Q Bull Northwest Univ Med Sch 30:213-214

8. Leedham PW, Baum M, Cullum PA (1971) Acute and modified rejection of heterotopic canine cardiac allotransplants studied by serial needle biopsy. Thorax 26:534-542

9. Mason JW (1978) Techniques for right and left ventricular endomyocardial biopsy. Am J Cardiol 41:887-892

10. Mills AS, Hastillo A, Thompson JA, Hess ML (1985) Expectations and limitations of endomyocardial biopsy. Can J Cardiol $1: 358-362$
11. Olmesdahl PJ, Gregory MA, Cameron EW (1979) Ultrastructural artefacts in biopsied normal myocardium and their relevance to myocardial biopsy in man. Thorax 34:82-90

12. Penn OC, McDicken I, Bos E, Nauta J (1975) Serial percutaneous biopsies from canine orthotopic cardic allografts. J Surg Res 18:615-618

13. Sakakibara S, Konno S (1962) Endomyocardial biopsy. Jpn Heart J 3:537-543

14. Salerno TA, Lince DP, Wasan SM, Charrette EJ (1979) Intraoperative transmural myocardial biopsy: a simple technique. Can J Surg 22:487

15. Shu LG, de Geest H, Kesteloot H, van de Werf F, Aubert A, Borgers M, Janssen PA (1986) Closed chest myocardial biopsies: clinical uses and related problems. Acta Cardiol 41:79-87

16. Stengl M, Ramakers C, Donker DW, Nabar A, Rybin AV, Spatjens RLHMG, van der Nagel T, Wodzig WKWH, Sipido KR, Antoons G, Moorman AF, Vos MA, Volders PGA (2006) Temporal patterns of electrical remodeling in canine ventricular hypertrophy: focus on IKs downregulation and blunted beta-adrenergic activation. Cardiovasc Res 72:90-100

17. Tagawa H, Koide M, Sato H, Zile MR, Carabello BA, Cooper G 4th (1998) Cytoskeletal role in the transition from compensated to decompensated hypertrophy during adult canine left ventricular pressure overloading. Circ Res 82:751-761

18. Veinot JP (2002) Diagnostic endomyocardial biopsy pathologygeneral biopsy considerations, and its use for myocarditis and cardiomyopathy: a review. Can J Cardiol 18:55-65

19. Veinot JP (2002) Diagnostic endomyocardial biopsy pathology: secondary myocardial diseases and other clinical indications-a review. Can J Cardiol 18:287-296

20. Verduyn SC, Jungschleger JG, Stengl M, Spätjens RLHMG, Beekman JD, Vos MA (2004) Electrophysiological and proarrhythmic parameters in transmural canine left-ventricular needle biopsies. Pflügers Arch 449:115-122

21. Zenati MA, Bonanomi G, Chin AK, Schwartzman D (2003) Left heart pacing lead implantation using subxiphoid videopericardioscopy. J Cardiovasc Electrophysiol 14:949-953 